\title{
El efecto de la distancia al mercado sobre la pobreza rural en la Región Metropolitana de Santiago
}

Rodrigo Pérez. Organización de las Naciones Unidas para la Alimentación y la Agricultura (FAO), Santiago, Chile.

Alejandro Salazar. Pontificia Universidad Católica de Chile, Santiago, Chile.

William Foster. Pontificia Universidad Católica de Chile, Santiago, Chile.

Pablo Osses. Pontificia Universidad Católica de Chile, Santiago, Chile.

RESUMEN | La pobreza en Chile ha disminuido notablemente desde 1990. En el año 2006, la incidencia de la pobreza rural cayó bajo la de áreas urbanas, debido en parte a la movilidad poblacional. No obstante, los pobres rurales mantienen niveles educacionales y de ingresos bajos, lo que se explica, en parte, por el mayor aislamiento y el menor acceso que tienen a los mercados. Teniendo eso en consideración, este estudio sostiene que la distancia a áreas urbanas (en tiempo de viaje) se encuentra correlacionada con la pobreza, una vez que se controla por otros factores. Usando un modelo econométrico que relaciona información geográfica y socioeconómica de la Región Metropolitana, se concluye que el nivel de pobreza en un sector censal aumenta con la distancia, y que para una determinada distancia, la tasa de pobreza es menor si aumenta el acceso a medios de movilización, al mitigar el efecto de distancia.

PALABRAS CLAVE | pobreza, distribución espacial, relación campo-ciudad.

ABSTRACT | The national poverty headcount in Chile has declined considerably since 1990. In 2006, rural poverty rate fell below that of urban areas, due in part to population mobility. Rural areas, however, are still characterized by low educational levels and incomes, explained, in part, by low population density, remoteness to services, and limited access to markets for rural-produced products. This study finds that distance (measured as travel time) of rural populations to urban areas is associated with the incidence of poverty in rural communities after controlling for other factors. Using an econometric model based on geographical and socioeconomic information of the Metropolitan Region of Santiago, the study finds that poverty levels in census tracts increase with distance to Santiago, and, for a given distance, access to transportation reduces poverty, through a mitigation of the distance effect.

KEY WORDS | poverty, spatial distribution, countryside-city relationship.

Recibido el 16 de agosto de 2010, aprobado el 23 de septiembre de 2011

E-mail: Rodrigo Pérez, rodrigo.perez@fao.org | Alejandro Salazar, asalazab@uc.cl | William Foster, wfoster@uc.cl | Pablo Osses, posses@uc.cl

La publicación se enmarca dentro del Proyecto Fondecyt Nº 1100999 : "La nueva ruralidad funcional (densidad y tiempo de viaje); relación con las actividades económicas, recursos naturales y pobreza en las regiones metropolitanas de Chile. Comparación entre Regiones de Valparaíso, Biobío y Metropolitana de Santiago". Investigador responsable: Alejandro Salazar B. 


\section{Introducción}

El desempeño económico de Chile, en términos de crecimiento económico, de aumento en los niveles de ingreso y superación de la pobreza ${ }^{1}$, ha sido notable. Entre los años 1975 y 2007 el PIB per cápita se incrementó a tasas superiores al 5\% anual, el ingreso promedio ha subido en cerca de $150 \%$ real entre 1992 y 2006, mientras que la pobreza ha disminuido fuertemente hasta $13,7 \%$ (un retroceso de 24,9 y 5 puntos porcentuales respecto de 1990 y 2003, respectivamente). Por su parte, de acuerdo con la información de la Encuesta de Caracterización Socioeconómica Nacional (Casen), la pobreza en la Región Metropolitana de Santiago (RM) ha pasado desde una incidencia de 33\% hasta cerca del 10\% en el lapso de diecisiete años.

En el medio rural, los resultados son bastante similares a los obtenidos de forma general, e incluso la reducción de las tasas de pobreza (total y per cápita) es mayor que la que presenta el medio urbano. Entre 1990 y 2006, la pobreza disminuyó 24,6 puntos porcentuales hasta representar al $13,9 \%$ de la población en áreas urbanas (una tasa anual de reducción de $6 \%^{2}$ ) y 26,5 puntos porcentuales, hasta $12,3 \%$, en áreas rurales (con una tasa anual de 6,8\%). En la RM la reducción ocurrió a tasas de $5,6 \%$ y $6,6 \%$ en el medio urbano y rural, respectivamente.

Pese a lo anterior, la pobreza — especialmente la pobreza extrema - sigue siendo más marcada en áreas rurales y, de acuerdo con Pérez (2008), una parte importante de la reducción de la pobreza rural se debería a efectos migratorios desde zonas rurales a urbanas, particularmente de las personas más jóvenes (entre 25 y 35 años). Asimismo, diversos estudios demuestran la existencia de menores oportunidades y empleos en áreas rurales o remotas, por el aislamiento relativo respecto de las áreas urbanas, lo que tiende a generar mayores niveles de pobreza. En particular, Partridge y Rickman (2008) demuestran la existencia de una relación positiva entre la distancia a grandes áreas urbanas y la pobreza para EE.UU., como consecuencia de la atenuación de los efectos de las economías de aglomeración. El área metropolitana de Santiago de Chile representa, en tal sentido, un buen caso de estudio para evaluar la existencia de economías de aglomeración y para advertir si es que tales efectos disminuyen como consecuencia de la distancia al área urbana. Si bien el presente estudio no pretende ser comprehensivo en estos aspectos, busca entregar elementos de análisis y de discusión sobre los potenciales impactos que tendría en la pobreza la distancia al área urbana principal.

El concepto de "economías externas de escala" —o economías de aglomeración- fue introducido por Marshall (1890), aunque su desarrollo teórico (desde donde se desprende su nuevo auge) provino luego de los trabajos de Fujita en 1988, quien demostró los efectos de la aglomeración de las actividades económicas (Johansson \& Quigley, 2004). Los inicios de tal concepción se encuentran, sin embar-

1 Por pobreza se entenderá, para la primera parte de este documento, a las personas clasificadas como indigentes y pobres no indigentes según la encuesta Casen.

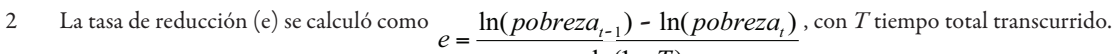
$\ln (1+T)$ 
go, en el modelo de Von Thünen, desarrollado en 1826, en el que se muestran las actividades agrícolas en anillos concéntricos a partir de un centro único. La idea principal de la teoría de Von Thünen era que dichas actividades se localizaban de acuerdo con la renta que los productores percibían de la tierra y los costos de transporte que enfrentaban (Figura 1). Este modelo, luego retomado en las últimas décadas por varios autores, ha sido la base para los actuales modelos de localización ya no solo referidos a actividades agrícolas, sino también a otros tipos de actividades ${ }^{3}$. En la Figura 1, por ejemplo, solo las actividades más rentables (R1 en el ejemplo) podrían ubicarse a una distancia como D1, y aquellas menos rentables (R3) se ubicarían en lugares más alejados del centro (como D3), puesto que, siendo menor la rentabilidad, los ingresos son menores.

\section{FIGURA 1 Distancia a la ciudad y renta de la actividad}

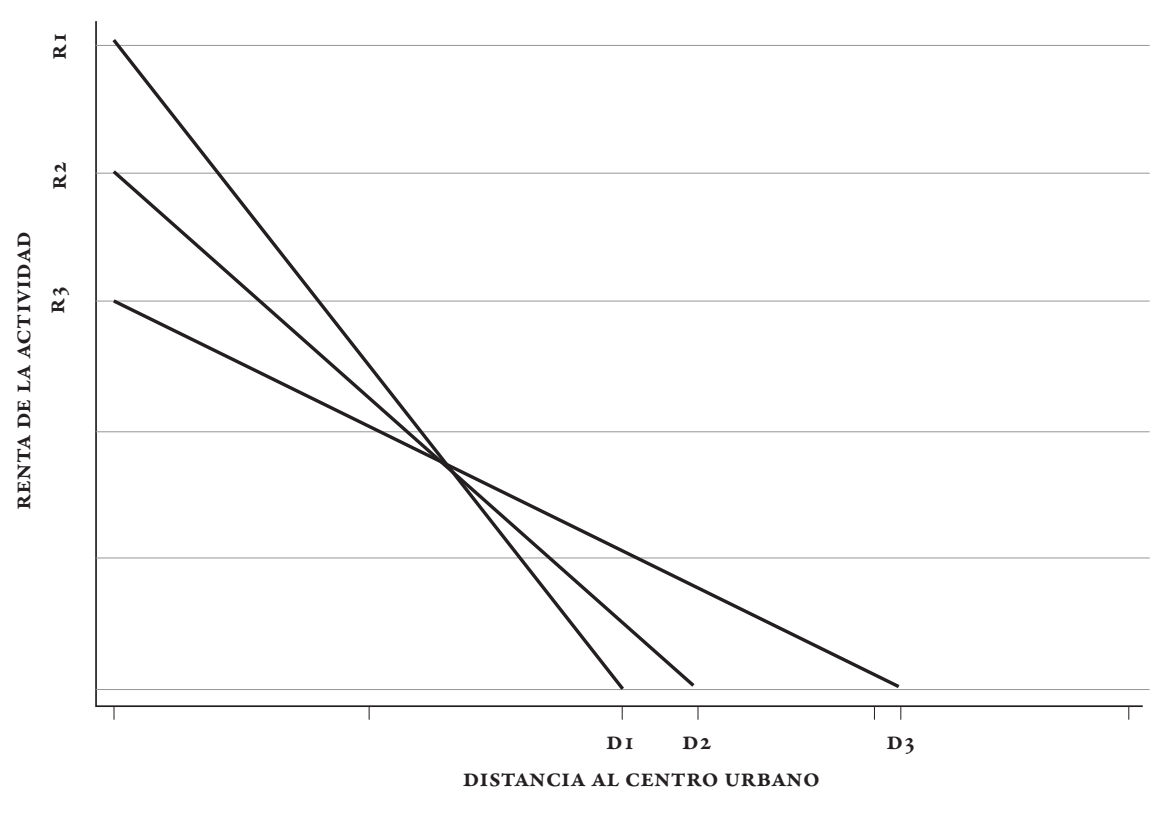

fuente elaboración propia, basado en Fujita, Krugman y Venables (2001).

Con relación a lo anterior, Alonso (1964) y Muth (1969) — que retoman el modelo de Von Thünen- establecen la existencia de un gradiente de precios del suelo que tiene su punto más alto en el centro de la ciudad y decrece hacia la periferia, de forma tal que las actividades económicas que se localicen más cerca del centro o distrito financiero (CBD, central business district) deberán pagar más en función de disminuir los costos de transporte hasta dicho CBD. De esta manera, y

Para mayor detalle véase, por ejemplo, Fujita et al. (2001), y Baldwin, Forslid, Martin, Ottaviano y Robert-Nicoud (2003). 
suponiendo que a los diferentes actores económicos les conviene encontrarse cerca del centro - debido a los beneficios de las economías de aglomeración, tales como la posibilidad de acceder a mercados, la disminución de los tiempos y costos de transporte, la velocidad de transmisión de nuevas ideas y conocimiento, etcétera-, entonces las actividades se distribuirán de forma tal de encontrar solo a las más rentables cerca del CBD, y luego se extenderán en un gradiente de actividades cada vez menos rentables en la medida en que se alejan del CBD.

De esta forma, las grandes aglomeraciones generarían economías de escala que permitirían tanto una diversificación de las actividades económicas (existiendo más oportunidades que en otros espacios), como un mayor nivel de ingreso asociado a las sinergias antes comentadas. En tal sentido, los ingresos y las oportunidades de empleo de los trabajadores aumentarían en función del tamaño de las ciudades y disminuirían en función de la distancia a las ciudades (Henderson, 2000; Johansson \& Quigley, 2004). A causa de lo anterior, las urbes generarían una atracción para los trabajadores, ya que representarían una oportunidad de mejoramiento de los ingresos y de mayor acceso a servicios (hospitales, escuelas, etcétera) que no pueden ser abastecidos en áreas remotas. Esta situación ha sido evidenciada por Ravallion, Chen y Sangraula (2008), Stark y Fan (2007), y Partridge y Rickman $(2008)^{4}$.

De igual forma, Köbrich, Villanueva y Dirven (2004) señalan la importancia del acceso a los mercados como un factor relevante para explicar altos niveles de pobreza. De acuerdo con los autores, solo algunos de los hogares pobres están en condiciones de acceder a los mercados - lo que involucra el acceso a la compra y venta de bienes, mano de obra y capital, así como a la información-. Finalmente se señala que algunos de los criterios para determinar la condición de aislamiento respecto de los mercados y sus consecuencias sobre la pobreza son el ingreso y la educación; la existencia de la infraestructura vial, de comunicaciones, de transporte público, de mercados o plazas para comprar y vender; el acceso a crédito y a servicios, entre otros.

El objetivo principal del estudio es encontrar evidencia sobre el efecto que tiene la distancia - como una aproximación al aislamiento- sobre la pobreza en las áreas que circundan el área urbana de Santiago. La hipótesis es que la distancia, medida como tiempo de viaje desde cada sector censal hasta la mancha urbana de Santiago ${ }^{5}$, se encuentra correlacionada con la pobreza de la población de un sector censal, una vez que se toman en cuenta variables sociales, económicas, geográficas y demográficas. En tal sentido, si se controla por variables sociales y económicas de contexto, resultaría más probable ser pobre en la medida en que se esté más lejos del mercado principal. Se considera que el principal aporte de este documento es la

4 Los efectos negativos de las aglomeraciones (deseconomías de escala), los cuales no serán estudiados en este documento, se encuentran asociados usualmente a alta congestión, y también a otros elementos, como la contaminación, que podrían reducir el bienestar de la población. Una mayor y mejor discusión teórica al respecto se encuentra, aun cuando no exclusivamente, en Fujita et al. (2001) y en Henderson (2005). De igual forma, para una aproximación mayor a la realidad latinoamericana, se sugiere revisar el texto de la Comisión Económica para América Latina y el Caribe (Cepal, 2009).

5 Específicamente, al mercado central de Santiago. 
sistematización y el uso de información censal para la estimación, con un modelo econométrico, de la existencia de correlaciones entre la distancia al centro urbano y la pobreza rural ${ }^{6}$.

El estudio se encuentra estructurado en cuatro secciones, incluyendo la introducción. La segunda sección explica la información y el modelo utilizado para la contrastación de la hipótesis principal. La tercera muestra y discute los resultados obtenidos, mientras que la última concluye en relación con los mismos, además de entregar algunas recomendaciones para posteriores estudios.

\section{Materiales y método}

Para el desarrollo de este estudio se utilizó la información del Censo de Población y Vivienda del año 2002 y datos geográficos (tiempo de viaje y densidad) a nivel de sectores censales ${ }^{7}$. Los datos geográficos de detalle utilizados fueron originados, desarrollados e integrados en el marco del proyecto Fondecyt $\mathrm{N}^{\circ} 1100999$ : "La nueva ruralidad funcional (densidad y tiempo de viaje); relación con las actividades económicas, recursos naturales y pobreza en las regiones metropolitanas de Chile. Comparación entre regiones de Valparaíso, Biobío y Metropolitana de Santiago". Igualmente, la información socioeconómica y demográfica fue procesada e integrada al análisis espacial a partir del Censo de Población y Vivienda 2002, utilizando el software Redatam + SP.

Es importante destacar que la información socioeconómica del censo no se encuentra a nivel de hogares o personas (como por ejemplo sí es posible de encontrar en el caso de las encuestas de hogares, como la Casen en Chile), sino que es entregada en forma de frecuencias a escala de sectores censales y, por tanto, no permite relacionar a una determinada persona u hogar con ciertas características observables. Sin embargo, sí permite establecer sectores (censales) de análisis que son relacionables con características económicas y sociales diversas al interior del sector censal.

En este estudio se utilizaron sectores censales que corresponden a pequeñas agrupaciones de hogares localizados en áreas rurales y que conforman, a criterio del INE, una unidad censal. Estas agrupaciones no tienen una extensión ni composición uniforme, pudiendo existir sectores con grupos de menor o mayor población, e igualmente sectores de mayor o menor extensión física. Esto, como se verá más adelante, pudiera tener impactos sobre la variable "densidad del sector censal" razón por la que se realiza un ajuste considerando la densidad comunal ${ }^{8}$. Finalmente,

6 Debe tenerse en cuenta que para efectos del presente estudio, para acotar aquello que se considera rural se utiliza la definición de sectores censales rurales tal como vienen especificados por la información del Censo de Población y Vivienda 2002, por parte del Instituto Nacional de Estadísticas (INE). Discusiones y definiciones más complejas de este fenómeno pueden encontrarse en el documento de Chomitz, Buyz y Thomas (2004), las que también fueron tomadas en el documento del Banco Mundial a cargo de De Ferranti, Perry, Foster, Lederman y Valdés (2005).

7 Los sectores censales son definidos por el INE y espacializados y socioestadísticamente caracterizados por Salazar y Osses (2008), y Salazar, Hidalgo y Paéz (2011).

8 La densidad de un sector es definida como la razón entre el número personas de un sector y el tamaño del mismo. El tamaño de un sector, no obstante, no corresponde a límites administrativos, sino que es definido por el INE delimitando áreas de concentraciones de población, quedando fuera las áreas sin población. Esto puede producir que existan sectores altamente densos en áreas relativamente despobladas (comunas muy poco densas), con un número muy bajo de población pero en un espacio pequeño y concentrado. Esta es la razón por la que se utiliza el ponderador de la densidad de la comuna como variable auxiliar de análisis y de corrección de posibles sesgos. 
debe destacarse que los sectores censales son la menor representación física de una unidad de hogares en áreas rurales.

El modelo empleado considera variables socioeconómicas (edad, escolaridad, pertenencia a culturas indígenas y categoría ocupacional) y geográficas (densidad del sector censal, densidad comunal, número de personas por sector y tiempo de viaje hasta Santiago). Además se considera relevante, por ser factor de aumento de acceso, el poseer un automóvil; sin embargo, esta variable se encuentra presumiblemente relacionada con el tiempo de viaje y con la pobreza, por lo que se implementó como una variable instrumental, evitando así problemas de endogeneidad (véase resultados del test de Hausman en el Cuadro 4).

El modelo empleado es, por tanto, uno de mínimos cuadrados en dos etapas (2SLS), donde se relaciona la tasa de pobreza ${ }^{9}$ en un sector censal con diversas características sociales y económicas, las que se encuentran explicadas en el Cuadro 1. En particular, el modelo establece como variable dependiente la de porcentaje de personas pobres respecto del total de población en el sector (Pov) como una función de una variable que mide el número de personas mayores de sesenta años en el sector ( $3 \mathrm{raEdad}$ ), una variable que identifica a las personas que declaran pertenecer a alguna etnia (Etnia), una variable que contabiliza el número de personas asalariadas en el sector censal (Asalariado), una variable que mide la tasa de familiares no remunerados en el sector (FamNoRem), una variable que identifica a las personas que no han asistido nunca a la escuela formal (SinEsc), una variable que representa a las personas que cuentan con una educación superior a la secundaria (Esc13más), una variable que mide el tiempo de viaje desde un sector censal hasta el área urbana de Santiago (TiempoViaje), una proxi de aglomeración en cada sector (NúmeroPersonas), la densidad del sector censal ponderada por la densidad comunal (Densidad $W$ ), la variable que mide la interacción entre ambas, la densidad del sector censal y el tiempo de viaje (Densidad*TiempoViaje).

Finalmente, se consideró también la inclusión de una variable que mida la proporción de los hogares que poseen un medio de movilización (Auto). Sin embargo, esta variable (incluida directamente de forma simple) tiene una alta probabilidad de ser endógena (simultáneamente determinada con los niveles de pobreza); es decir, que factores que influyen en la perturbación del modelo que explica niveles de pobreza están probablemente correlacionados también con la probabilidad de tener un medio de movilización. Por esta razón se construyó una variable instrumental a partir de otras variables independientes que permiten su estimación, para luego ser incluida en el modelo de forma indirecta, como una probabilidad de tener auto y no la tenencia en sí del mismo. Para instrumentalizarla se consideraron las

9 Para el modelo final se consideró pobre a las personas que pertenecen a los grupos socioeconómicos d y e; sin embargo, igualmente se realizaron estimaciones definiendo como pobre solamente al grupo e, y se verificó que los resultados y las conclusiones no varían de forma considerable. Resulta importante mencionar que la razón de utilizar este concepto de pobreza responde exclusivamente a criterios prácticos, relacionados con la fuente de información original. Aun así, es de la mayor relevancia destacar que la pobreza es un fenómeno multidimensional, que debe ser estudiado en diversos aspectos, dentro de los cuales se incluyen factores sociales, de contexto, económicos propiamente tales y culturales. $\mathrm{Al}$ respecto resulta interesante consultar, por ejemplo, la información generada por el Consejo Nacional de Evaluación de la Política de Desarrollo Social de México (Coneval) y el trabajo efectuado por organismos internacionales como Cepal y FAO, la Organización de las Naciones Unidas para la Alimentación y la Agricultura. Para los fines del presente documento se trabajará de forma exclusiva con la definición anteriormente señalada. 
variables de tercera edad, etnia, tipo de trabajador, nivel de escolaridad, tiempo de viaje, número de personas, la densidad, estado civil, lugar de trabajo o estudio, sexo $\mathrm{y}$ algunas interacciones entre las mismas variables.

El Cuadro 1 resume la información de las variables utilizadas en el modelo, mientras que el Cuadro 2 entrega estadística descriptiva de las mismas.

CUADRO 1 Lista y descripción de variables incluidas en el modelo

\begin{tabular}{|c|c|c|}
\hline VARIABLE & NOMBRE & DESCRIPCIÓN \\
\hline Pov & Pobreza & $\begin{array}{l}\text { Razón entre pobres (grupos socioeconómicos d y/o e) y } \\
\text { total de población del sector censal. }\end{array}$ \\
\hline Auto & Auto & Número de autos por persona en un sector. \\
\hline 3raEdad & Tercera edad & $\begin{array}{l}\text { Número de personas mayores de } 60 \text { años respecto del total } \\
\text { de personas en un sector censal. }\end{array}$ \\
\hline Etnia & Etnia & $\begin{array}{l}\text { Personas que pertenecen a alguna cultura indígena respecto } \\
\text { del total de personas en el sector censal. }\end{array}$ \\
\hline Asalariado & Asalariado & $\begin{array}{l}\text { Número de ocupados dentro de la categoría asalariados } \\
\text { respecto del total de personas. }\end{array}$ \\
\hline FamNoRem & Familiar no remunerado & $\begin{array}{l}\text { Número de ocupados dentro de la categoría familiar no } \\
\text { remunerado respecto del total de personas. }\end{array}$ \\
\hline SinEsc & Sin escolaridad & Número de asalariados respecto del total de personas. \\
\hline Esc13más & Escolaridad $\geq 13$ & $\begin{array}{l}\text { Personas que poseen } 13 \text { años o más de escolaridad respecto } \\
\text { del total de personas del sector. }\end{array}$ \\
\hline TiempoViaje & Tiempo de viaje & $\begin{array}{l}\text { Tiempo de viaje desde el centro de Santiago hasta el centro } \\
\text { de un sector censal, medido en horas. }\end{array}$ \\
\hline NúmeroPersonas & Número de personas & Número total de personas dentro de un sector censal. \\
\hline DensidadW & Densidad ponderada & $\begin{array}{l}\text { Densidad del sector censal (habitantes por } \mathrm{km}^{2} \text { ) ponderada } \\
\text { por la densidad comunal promedio. }\end{array}$ \\
\hline Densidad*TiempoViaje & $\begin{array}{l}\text { Densidad y tiempo de } \\
\text { viaje }\end{array}$ & $\begin{array}{l}\text { Variable que mide el efecto interactivo de la densidad del } \\
\text { sector censal y el tiempo de viaje. }\end{array}$ \\
\hline
\end{tabular}

FUENTE ELABORACIÓN PROPIA 
CUADRO 2 Estadística descriptiva de las variables utilizadas en el modelo

\begin{tabular}{|c|c|c|c|c|c|}
\hline Variable & $\mathrm{N}$ & Media & Desv. estándar & Mínimo & Máximo \\
\hline Auto & 4064 & 0,1560885 & 0,162344 & 0 & 3 \\
\hline 3raEdad & 4064 & 0,1159408 & 0,1304696 & 0 & 1 \\
\hline Etnia & 4064 & 0,0168359 & 0,0579201 & 0 & 1 \\
\hline Asalariado & 4064 & 0,2834893 & 0,1548377 & 0 & 1 \\
\hline $\begin{array}{l}\text { Familiar No } \\
\text { Remunerado }\end{array}$ & 4064 & 0,0072608 & 0,0366528 & 0 & 1 \\
\hline Escolaridad $=0$ años & 4064 & 0,0808933 & 0,1080802 & 0 & 1 \\
\hline Escolaridad $\geq 13$ años & 4064 & 0,1491383 & 0,1694962 & 0 & 1 \\
\hline Tiempo de Viaje & 4064 & 0,7273058 & 0,4195316 & 0,0853705 & 3,912305 \\
\hline Número de Personas & 4064 & 36,08022 & 69,53812 & 1 & 1463 \\
\hline Densidad [W] & 4064 & 4,924222 & 24,77339 & 0,0010096 & 898,6367 \\
\hline $\begin{array}{l}\text { Densidad*Tiempo } \\
\text { de Viaje }\end{array}$ & 4064 & 167,432 & 350,9291 & 0,0933083 & 8952,325 \\
\hline
\end{tabular}

FUENTE ELABORACIÓN PROPIA

NOTA LAS VARIABLES UTILIZADAS SON TASAS, ES DECIR, CORRESPONDEN AL NÚMERO DE VECES QUE SE OBSERVA LA VARIABLE EN UN SECTOR CENSAL DIVIDIDO POR EL NÚMERO TOTAL DE OBSERVACIONES DENTRO DEL SECTOR. EN EL CASO DE LA VARIABLE 'AUTO', EL VALOR MÁXIMO INDICA QUE, AL MENOS EN UN SECTOR, SE CONTABILIZA EL TRIPLE DE AUTOS QUE DE PERSONAS. 


\section{Resultados}

Un resultado preliminar, previo al modelo econométrico, relaciona la variable "tiempo de viaje" con el porcentaje de población pobre respecto del total de población pobre de la muestra completa. Este análisis permite conocer la distribución espacial de la población pobre en la RM y evidenciar si es que, en un análisis exploratorio, es posible encontrar correlaciones positivas inmediatas entre tiempo de viaje y pobreza, que hagan innecesaria la especificación de un modelo de prueba.

Los datos del Censo de Población y Vivienda de la RM muestran que no es evidente que la población más pobre viva en áreas alejadas a Santiago. En particular, tal como lo muestra la Figura 2, los grupos poblacionales pobres tendrían una distribución espacial concentrada a alrededor de una hora de Santiago; sin embargo, decaerían después de ese punto hasta casi desaparecer más allá de dos horas de distancia.

En este sentido, no es posible concluir que la población más pobre se concentra en áreas alejadas de la ciudad si es que no se consideran otras variables de control. En este análisis exploratorio se concluye que la población pobre se concentra a distancias (medidas como tiempo de viaje) relativamente cercanas a la ciudad y tiende a desaparecer lejos de la urbe principal; no obstante, esta relación no toma en consideración otras variables de contexto, que pudieran afectar los resultados preliminares. Ello hace necesario, en función de encontrar tal correlación entre distancia y pobreza, la implementación de un modelo que permita establecer mayores niveles de correlación entre estas variables al tomar en cuenta otras variables de control.

\section{FIGURA 2 Distribución espacial de la población pobre en la RM}

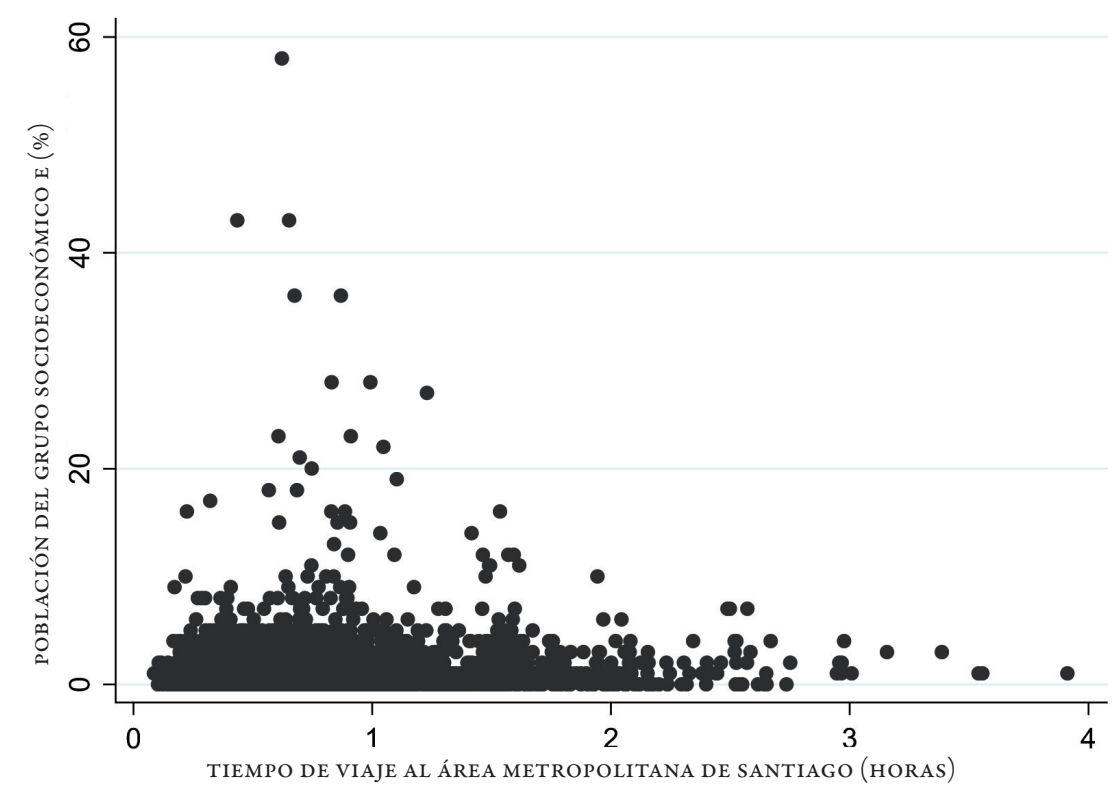

FUENTE ELABORACIÓN PROPIA CON BASE EN EL CENSO 2002. 
El segundo paso consiste, entonces, en evaluar la existencia de correlaciones, mediante el modelo propuesto. Debido a que los valores se encuentran en tasas (excepto en el caso de las variables geográficas) —es decir, el número de personas que cumplen con determinada característica en un sector, respecto del total de personas del sector-, la relación entre la variable dependiente y las demás se da en términos de porcentajes o elasticidades.

Como se señaló de forma previa, se consideró pobre a las personas de los grupos socioeconómicos d y e, por lo que los resultados están referidos a esa población. Sin embargo, se realizaron las mismas estimaciones considerando pobre solo al grupo socioeconómico e, de forma tal de darle robustez al estudio. A continuación se detallan los resultados referidos a la primera estimación (grupos d y e), encontrándose en el Cuadro 3 tanto los detalles de esta estimación como los de aquella que solo considera al grupo e.

Los resultados del Cuadro 3 indican que la población perteneciente a culturas indígenas (etnias) está correlacionada de forma positiva con mayores tasas de pobreza. En particular, el 1\% más de población indígena se traduce en un aumento de $0,09 \%$ en la pobreza de un sector determinado. La elasticidad de los trabajadores familiares no remunerados respecto de la pobreza es de $0,17 \%$; es decir, que a medida que aumentan los trabajadores no remunerados, la pobreza aumenta consecuentemente. El efecto de la población de la tercera edad es notorio. El incremento de un $1 \%$ de la población mayor de sesenta años genera un aumento de $0,15 \%$ en la tasa de pobreza.

En el caso de los asalariados, la elasticidad respecto de la pobreza es de $-0,04 \%$. Este resultado señala que un aumento en la tasa de personas asalariadas produce una reducción en la incidencia de la pobreza. Resulta interesante notar que la variación del $1 \%$ en el número de personas con un nivel de escolaridad superior o igual a trece años - es decir, con estudios superiores a los de enseñanza secundaria- hace caer la pobreza en $-0,08 \%$, en tanto que la elasticidad relativa a quienes nunca han asistido a la escuela y la pobreza es de $0,8 \%$. En tal sentido, aun cuando el impacto generado por el incremento del nivel de escolaridad parece limitado en términos de reducción de la pobreza, el tener población sin educación alguna presenta una correlación cercana a 1 respecto de la pobreza.

En este estudio se planteó expresamente como hipótesis la existencia de una relación directa entre tiempo de viaje y pobreza, y de forma implícita se relevó la importancia de la densidad como factor de aglomeración y de superación de la pobreza. En el segundo caso, aun cuando la relación es tal como se previó, es posible señalar que la densidad relativa — es decir, ponderada por la densidad comunaltiene un aporte escaso en la reducción de la pobreza (una elasticidad de -0,0002\%, por lo que solo un incremento de $100 \%$ en la densidad ponderada reduciría en $0,02 \%$ la pobreza de un sector censal). ${ }^{10}$ El tiempo de viaje, en cambio, tiene un

10 No obstante, este resultado puede estar influido por lo descrito en la metodología acerca del tamaño de los sectores censales y el error al que puede conducir tal medición. 
aporte no menor en el incremento de la pobreza: una hora de viaje más se traduce en un incremento de $0,06 \%$ en la tasa de pobreza al interior de la RM. Este dato resulta relevante, puesto que, aun cuando al interior de la RM existe una alta conectividad y un gran mercado (Santiago), la pobreza se correlaciona positivamente con la distancia.

Un incremento del $1 \%$ en el número de personas presentes en un sector, variable que puede ser tomada como una proxy de densidad, hace caer la tasa de pobreza en $0,005 \%$. Igualmente, el aporte de la variable que captura la interacción entre la densidad y el tiempo de viaje a la reducción de la pobreza, es cercano a cero.

Finalmente, el aumento del $1 \%$ en la tasa de motorización ${ }^{11}$ hace caer la tasa de pobreza en $0,9 \%$. Al igual que en el caso de la escolaridad, esta variable presenta una correlación cercana a -1 con la pobreza. En tal sentido, el aporte que implica aumentar la tasa de motorización en los lugares rurales apartados, de forma de que estos puedan acceder a los mercados ( $\mathrm{y}$ a servicios asociados con las grandes aglomeraciones), es casi directo. Este es uno de los resultados más importantes del estudio, ya que puede considerarse que elimina, al menos en parte, el efecto de la distancia pura, al reducir el tiempo de viaje de las personas en áreas rurales. Es importante mencionar que se trata de la instrumentalización de la variable "transporte" y no de la tenencia en sí de un automóvil. En tal sentido, la variable considerada representa una proxy que corrige e indica que determinados grupos de personas tienen una mayor cercanía al centro urbano que la que sugiere su sola ubicación geográfica.

Resulta importante destacar que, bajo la consideración de pobreza como exclusiva del grupo socioeconómico e (segunda estimación del modelo, donde se excluye al grupo socioeconómico d), los resultados no se modifican de forma considerable en ninguna de las variables de relevancia (tiempo de viaje, tasa de motorización, número de personas, etcétera), aun cuando tienden a atenuarse en magnitud.

Como se señaló de forma previa, se asume que la tenencia de un automóvil se encuentra directamente relacionada con el nivel de ingreso, y este con la pobreza en un sector censal. Sobre esa base, se consideró instrumentalizar la variable "auto" de la forma que se menciona anteriormente en la metodología. Finalmente, para comprobar que la instrumentalización de la variable elimina el sesgo de endogeneidad del modelo, se considera correr el modelo con la variable pura, es decir, directamente, y luego con la variable instrumentalizada, para luego observar las diferencias entre ambos modelos. En caso de que la variable no debiera ser instrumentalizada, se esperaría que no existieran diferencias entre ambas estimaciones, lo que se prueba mediante el test de endogeneidad de Hausman. Como se observa en el Cuadro 4, las diferencias entre ambos modelos son significativas, rechazándose la hipótesis nula y corroborándose la necesidad de la instrumentalización de la variable "auto" y la estimación mediante el método de mínimos cuadrados en dos etapas (2SLS). 


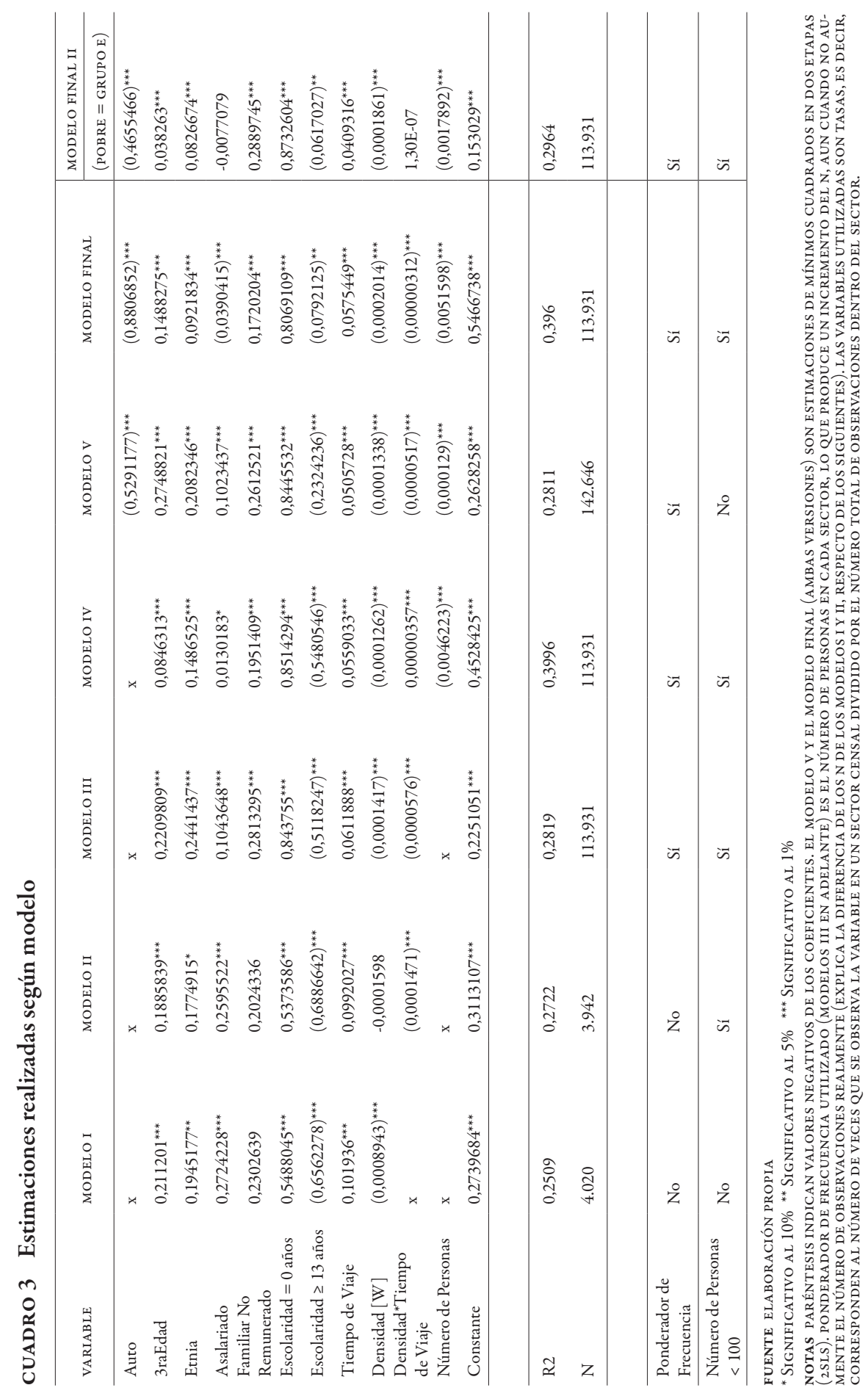


CUADRO 4 Test de Hausman para el modelo final

\begin{tabular}{|c|c|c|c|c|}
\hline & \multicolumn{2}{|c|}{ Coeficientes } & \multirow{3}{*}{$\begin{array}{c}(\mathrm{b}-\mathrm{B}) \\
\text { Diferencia }\end{array}$} & \multirow{3}{*}{$\begin{array}{l}\text { Sqrt(diag(V_b- } \\
\quad \text { V_B }) \\
\text { Error Estándar }\end{array}$} \\
\hline & & & & \\
\hline & 2SLS & OLS & & \\
\hline Auto & $-0,88069$ & $-0,42041$ & $-0,46027$ & 0,06426 \\
\hline 3raEdad & 0,148828 & 0,11528 & 0,033551 & 0,006331 \\
\hline Etnia & 0,092183 & 0,1217 & $-0,02951$ & 0,004233 \\
\hline Asalariado & $-0,03904$ & $-0,01183$ & $-0,02721$ & 0,004558 \\
\hline FamNoRem & 0,17202 & 0,1841 & $-0,01208$ & 0,01867 \\
\hline Escolaridad $=0$ & 0,806911 & 0,83018 & $-0,02327$ & 0,003568 \\
\hline Escolaridad $>=13$ & $-0,07921$ & $-0,32424$ & 0,245031 & 0,03489 \\
\hline Tiempo de viaje & 0,057545 & 0,05669 & 0,000858 & 0,000315 \\
\hline $\begin{array}{l}\text { Número de } \\
\text { Personas }\end{array}$ & $-0,00516$ & $-0,00488$ & $-0,00028$ & 4,06E-05 \\
\hline Densidad [W] & $-0,0002$ & $-0,00016$ & $-3,90 \mathrm{E}-05$ & $5,05 \mathrm{E}-06$ \\
\hline $\begin{array}{l}\text { Densidad*Tiempo } \\
\text { de Viaje }\end{array}$ & $-3,12 \mathrm{E}-06$ & $3,79 \mathrm{E}-07$ & $-3,50 \mathrm{E}-06$ & $5,01 \mathrm{E}-07$ \\
\hline
\end{tabular}

Chi2 (8) $=201.17$

Prob $>$ Chi $2=$

0.0000

FUENTE ELABORACIÓN PROPIA

\section{Discusión y conclusiones}

La pobreza, o la incidencia de la misma en un espacio geográfico dado, se encuentran determinadas por diversos factores, entre los que - como es de esperar- se consideran elementos sociales y económicos. Los activos de las personas, de los cuales acá solo se mencionan los relacionados con educación y la posesión de un medio de movilización propio, determinan de forma importante la existencia de pobreza.

En el estudio realizado se buscó encontrar evidencia que respalde el hecho de que la distancia, medida como el tiempo de viaje desde un sector censal hacia el área urbana de Santiago, o la falta de acceso, se encuentran correlacionadas con mayores niveles de pobreza. El primer hecho importante de destacar es que efectivamente existe un efecto relacionado con el tiempo de viaje y que señala que si este aumenta, la pobreza lo hace de forma similar. El estimador de la tasa de motorización, variable que mide la posibilidad de acceso a mercados y a la aglomeración (con las ventajas que esta ofrece), tiene una correlación inversa muy alta con incrementos en pobreza; o, dicho de otra forma, tiene una correlación elevada con la reducción de la pobreza en un sector censal determinado. La elasticidad de reducción de la pobreza medida por la tasa de motorización indica una correlación de esta de alrededor de -0,9. En tal sentido, al mismo tiempo que el tiempo de viaje por sí solo 
explica el aumento en la tasa de pobreza, la falta de acceso a los mercados es aún más importante como motor para su superación en la RM.

Los resultados indican que el efecto que la aglomeración de Santiago tiene sobre la persistencia o la reducción de la pobreza está directamente relacionado con los efectos de las economías de escala que generan las grandes urbes. Este efecto es incluso notorio en el caso de cada sector rural específico, donde a medida que aumenta el número de personas y la densidad, la tasa de pobreza disminuye. Este mismo fenómeno es apreciable, aun cuando de menor manera, en la relación con las tasas de densidad poblacionales.

En posteriores estudios resultaría relevante realizar estas estimaciones para otras regiones del país, a fin de evaluar la correspondencia con los resultados encontrados acá. De forma preliminar, la hipótesis es que mientras mayores sean las diferencias de tamaño entre la urbe y los sectores rurales aledaños, así como mayor sea el tiempo de viaje entre ambos lugares, mayor será la correlación entre distancia y pobreza. En el caso de la RM, el efecto de la distancia pudiera no ser tan alto como en otras regiones, debido principalmente a la gran cantidad de accesos con que cuenta el área urbana de Santiago, con carreteras de alta velocidad que permiten tanto la llegada de los pobladores rurales a mercados y servicios, como el efecto de la migración inversa (incluso diaria) de personas con alto poder adquisitivo que viven en áreas alejadas pero que trabajan en la urbe.

En resumen, pese a que la RM es quizá la región con mayor dinamismo del país, y a pesar de que cuenta con un gran número de caminos y carreteras, el efecto de distancia al mercado principal es aún relevante. En tal sentido, al controlar por otros factores, existirían procesos de desigualdad en la distribución de la riqueza no solo entre regiones, sino que dentro de ellas. Tal como se señaló previamente, debido a la hipótesis manejada en este estudio, se espera que este efecto se vea amplificado en otras regiones del país, tanto respecto de Santiago (diferencias interregionales) como respecto del centro regional principal (diferencias intrarregionales).

Entre las limitantes del estudio se encuentra el hecho de que solo se utilizaron sectores censales tal como vienen definidos por el INE. Para posteriores estudios resultaría interesante construir tales sectores de forma tal que tengan tamaños comparables. Al mismo tiempo, ello permitiría eliminar el sesgo generado por la variable "densidad", que contabiliza solo las personas que están dentro de un sector donde existen observaciones y, al hacerlo así, excluye áreas donde no hay habitantes. Esto podría tener efectos sobre las estimaciones, razón por la cual en el estudio se utilizó un ponderador de la densidad asociada a la densidad comunal promedio.

Finalmente, aunque se considera que la técnica desarrollada en este estudio representa un avance en la medición de la importancia que tienen las variables geográficas sobre distintas variables socioeconómicas, debe considerarse la posibilidad de realizar nuevas estimaciones, utilizando la técnica de matching entre el Censo de Población y Vivienda y las encuestas Casen, de forma tal de tener una mejor aproximación a las características de los habitantes de las áreas rurales y mejorar, de esta manera, las estimaciones realizadas. OEURE 


\section{Referencias bibliográficas}

Alonso, W. (1964). Location and Land Use. Cambridge, MA: Harvard University Press.

Aroca, P. (2007). Impacto sobre el crecimiento regional de la migración y conmutación interregional en

Chile. Taller Migración interna y desarrollo en Chile: diagnóstico, perspectivas y políticas. Comisión Económica para América Latina y el Caribe-Centro Latinoamericano y Caribeño de Demografía (Cepal-Celade), Santiago, Chile.

Baldwin, R., Forslid, R., Martin, P., Ottaviano, G. \& Robert-Nicoud, F. (2003). Economic Geography and Public Policy. Princeton, NJ: Princeton University Press.

Banco Interamericano de Desarrollo (BID). (2001). Informe anual sobre actividades de desarrollo rural. Washington, D.C.: BID, Divisiones de Medio Ambiente de los Departamentos Regionales y Unidad de Desarrollo Rural del Departamento de Desarrollo Sostenible.

Comisión Económica para América Latina y el Caribe (Cepal). (2007, noviembre). Panorama social de América Latina. Santiago, Chile: Cepal, División de Desarrollo Social y División de Estadística y Proyecciones Económicas.

Comisión Económica para América Latina y el Caribe (Cepal). (2009, marzo). Economía y territorio en América Latina y el Caribe. Desigualdades y políticas. Santiago, Chile: Autor.

De Mattos, C. \& Hidalgo, R. (2007). Santiago de Chile. Movilidad espacial y reconfiguración metropolitana. Santiago, Chile: Instituto de Estudios Urbanos y Territoriales, Instituto de Geografía, Pontificia Universidad Católica de Chile.

De Mattos, C., Riffo, L., Salas, X. \& Yáñez, G. (2007). Cambios socio-ocupacionales y transformación metropolitana: Santiago, 1992-2002. En C. de Mattos \& R. Hidalgo (Eds.), Santiago de Chile. Movilidad espacial y reconfiguración metropolitana (pp. 89-115). Santiago, Chile: Instituto de Estudios Urbanos y Territoriales, Instituto de Geografía, Pontificia Universidad Católica de Chile.

Escolano, S. \& Ortiz, J. (2007). Patrones espaciales de movilidad de la población: Algunos efectos en la sociogeografía del gran Santiago. En C. de Mattos \& R. Hidalgo (Eds.), Santiago de Chile. Movilidad espacial y reconfiguración metropolitana (pp. 53-68). Santiago, Chile: Instituto de Estudios Urbanos y Territoriales, Instituto de Geografía, Pontificia Universidad Católica de Chile.

Chomitz, K., Buys, P. \& Thomas, T. (2004). Quantifying the Rural/Urban Gradient in Latin America and the Caribbean. Washington, D.C.: The World Bank.

De Ferranti, D., Perry, G., Foster, W., Lederman, D. \& Valdés, A. (2005). Beyond the City. The rural contribution to development. World Bank Latin American and Caribbean Studies. Washington D.C.: The World Bank.

Fujita, M., Krugman, P. \& Venables, A. (2001). The Spatial Economy. Cities, regions and international trade. Cambridge, MA: The MIT Press.

Henderson, J. V. (2000). How Urban Concentration Affects Economic Growth. Policy Research Working Paper 2326. Washington, D.C.: The World Bank Development Research Group, Infrastructure and Environment.

Henderson, J. V. (2005). Urbanization and growth. En P. Aghion \& S. Durlauf (Eds.). Handbook of Economic Growth (vol. 1, pp. 1543-1591). Amsterdam: Elsevier.

Johansson, B. \& Quigley, J. (2004). Agglomeration and Networks in Spatial Economies. University 
of California Postprints, paper 460. Disponible en: http://repositories.cdlib.org/postprints/460.

Köbrich, C., Villanueva, L. \& Dirven, M. (2004). Pobreza rural y agrícola. Entre los activos, las oportunidades y las politicas: una mirada hacia Chile. Serie Desarrollo Productivo 144. Santiago, Chile: Cepal, Red de desarrollo agropecuario, Unidad de desarrollo agrícola, División de desarrollo productivo y empresarial, Proyecto "Elementos para el diseño de una estrategia para superar la pobreza rural", Cepal/Indap.

Krugman, P. (1991). Geography and Trade. Cambridge, MA: The MIT Press/Leuven, Bélgica: Leuven University Press.

Marshal, A. (1980). Principles of Economic (8a ed.). Londres: Macmillan.

Muth, R. (1969). Cities and Housing, Chicago, Il: University of Chicago Press.

Partridge, M. \& Rickman, D. (2008. Distance from urban agglomeration economies and rural poverty. Journal of Regional Science, 48(2), 285-310. doi:10.1111/j.1467-9787.2008.00552.x

Pérez, R. (2008). Tiempo de viaje y pobreza rural en la Región Metropolitana de Santiago de Chile. Tesis de Magister, Departamento de Economía Agraria, Facultad de Agronomía e Ingeniería Forestal, Pontificia Universidad Católica de Chile, Santiago, Chile.

Ravallion, S., Chen, S. \& Sangraula, P. (2008). New Evidence on the Urbanization of Global Poverty. World Bank Policy Research Paper 4199, abril 2007. Washington, D.C.: The World Bank Development Research Group.

Rodríguez, J. \& González, D. (2006). Redistribución de la población y migración interna en Chile: Continuidad y cambio según los últimos cuatro censos nacionales de población y vivienda. Revista de Geografia Norte Grande, 35, 7-28.

Salazar, A. (2007). La periurbanización en la recomposición de los espacios rurales: Santiago de Chile. En C. de Mattos \& R. Hidalgo (Eds.), Santiago de Chile. Movilidad espacial y reconfiguración metropolitana (pp. 207-225). Santiago, Chile: Instituto de Estudios Urbanos y Territoriales, Instituto de Geografía, Pontificia Universidad Católica de Chile.

Salazar, A., Hidalgo, R. \& Páez, P. (2011). "Ruralidad y expansión residencial: Movilidad y funcionalidad en el periurbano emergente del área metropolitana de Santiago de Chile”. En S. Lencioni, S. Vidal-Koppmann, R. Hidalgo, P. Pereira (Eds.), Transformaçóes sócio-territoriais nas metrópoles de Buenos Aires, SãoPaulo e Santiago. Brasil, Chile y Argentina (pp. 205-224). São Paulo: Faculdade de Arquitetura e Urbanismo, Universidade de São Paulo.

Salazar, A. \& Osses, P. (2008, agosto 1). La ruralidad en la Región Metropolitana de Santiago de Chile (2002): determinación y relación con los grupos socio-profesionales. Scripta Nova. Revista Electrónica de Geografía y Ciencias Sociales (Barcelona: Universidad de Barcelona), 12(270) (112).

Von Thünen, J. H. (1826). Der Isolierte Staat in Beziehung auf Landschaft und Nationalökonomie. Hamburg. Traducción al inglés de C. M. Wartenberg, con el título Isolated State. Editado y con una introducción de Peter Hall. Oxford, NY: Pergamon Press, 1966.

Williamson, J. (2006). Poverty Traps, Distance, and Diversity: The migration connection. National Bureau of Economic Research, Working Paper 12549. Disponible en: http://www.nber.org/ papers/w12549.

World Bank. (2008). World Development Report 2008. Agriculture for development. Washington, D.C.: Autor. 